\title{
PREOPERATIVE PHYSICAL FUNCTION INFLUENCES ON STAIR CLIMBING ABILITY 1 MONTHS AFTER TKA.
}

\author{
So Young Lee ${ }^{1}$, Bo Ryun Kim¹ Eun Young Han \\ 1'Department of Rehabilitation Medicine, Jeju National University Hospital, Jeju National \\ University College of Medicine, Jeju, Republic of Korea
}

Introduction: Knee osteoarthritis (OA) is the one of the most common significant disease that is a leading cause of pain and swelling of joint, functional disability and impairment to the quality of life.

Purpose: This study was undertaken to identify preoperative physical performance factors predictive of stair climbing ability 1 month following total knee arthroplasty.

Method: In this prospective cohort study, we assessed a total of 84 patients who underwent a primary unilateral total knee arthroplasty (TKA). The gait parameters, isometric knee extensor and flexor strength of both knees, range of motion (ROM) of flexion and extension of surgical knee, 6 minute walk test (6MWT), timed up-and-go test (TUG), stair climbing test (SCT), Western Ontario McMaster Universities Osteoarthritis Index (WOMAC), EuroQoL five dimensions (EQ-5D) questionnaires, and visual analog scale (VAS) of knee pain were assessed before and 1 month after TKA.

Results: In the bivariate analyses, the postoperative SCTascent had a significant positive correlation with the SCT-ascent, SCT-descent, TUG, preoperative age, and a significant negative correlation with the preoperative 6MWT, peak torque (PT) extensor of surgical knee, PT flexor of surgical knee, PT extensor of the non-surgical knee, PT flexor of the nonsurgical knee. The postoperative SCT-descent had a significant positive correlation with the SCT-ascent, SCT-descent, TUG, preoperative age, WOMAC function, and a significant negative correlation with 6-MWT, PT extensor of surgical knee, PT flexor of surgical knee, PT extensor of the nonsurgical knee, PT flexor of the nonsurgical knee.

Table 1. Demographic and disease-related characteristics of the subjects $(\mathrm{N}=84)$.

\begin{tabular}{|c|c|}
\hline Variables & Values \\
\hline Age (years) & $72.0 \pm 6.0$ \\
\hline Sex, males/females & $8(9.5) / 76(90.5)$ \\
\hline BMI $\left(\mathrm{kg} / \mathrm{m}^{2}\right)$ & $27.1 \pm 3.6$ \\
\hline \multicolumn{2}{|l|}{ K-L grades } \\
\hline Grade 3 & $21(25.0)$ \\
\hline Grade 4 & $63(75.0)$ \\
\hline \multicolumn{2}{|l|}{ Lesion side } \\
\hline Right & $60(71.4)$ \\
\hline Left & $24(28.6)$ \\
\hline \multicolumn{2}{|l|}{ Comorbidities } \\
\hline Hypertension & $65(77.4)$ \\
\hline Diabetes mellitus & $16(19.0)$ \\
\hline Spine disease & $9(10.7)$ \\
\hline Osteoporosis & $42(50.0)$ \\
\hline
\end{tabular}

In the linear regression analyses, the preoperative TUG and PT extensor of surgical knee were factors predictive of the postoperative SCT-ascent. In addition, the preoperative SCTdescent and age were factors predictive of the postoperative SCT-descent.

Conclusions: According to preoperative predictive factors, we can propose the pre- and postoperative rehabilitation strategies to improve stair climbing ability in the early stages following TKA.

Table 2. Correlation between the preoperative physical performance and postoperative stair climbing ability

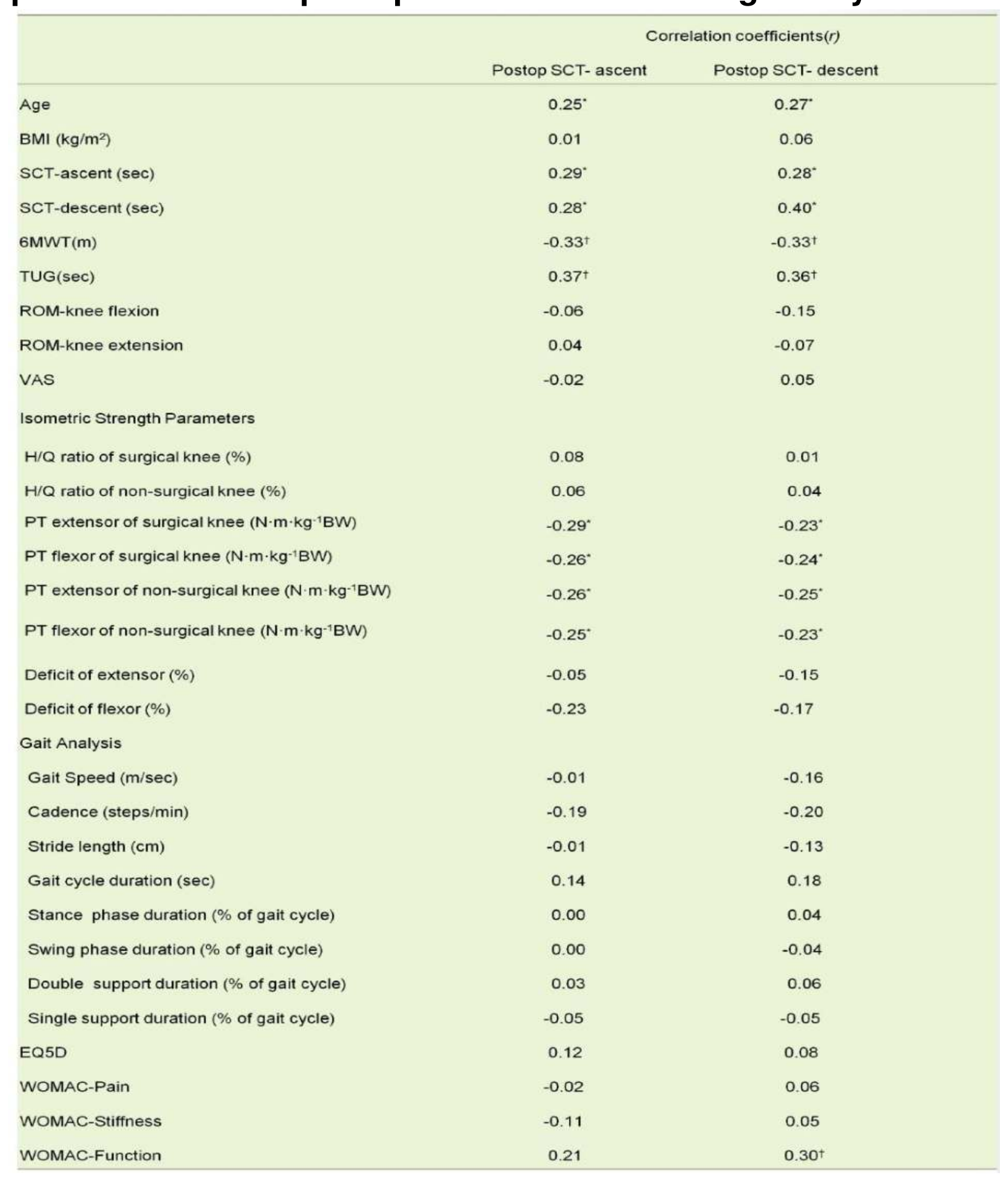

Table 3. Preoperative factors correlated with postoperative stair climbing ability by multivariate linear regression analysis

\begin{tabular}{lccc}
\hline \multicolumn{1}{|c}{ Outcome/Independent predictor } & Standardized (B) & $p$-value & Adjusted $R^{2}$ \\
\hline Postop SCT - ascent & & & \\
Preop TUG(sec) & 0.28 & $<0.01^{\dagger}$ & 0.18 \\
PT extensor of the surgical knee & -0.23 & $0.03^{*}$ & \\
Postop SCT - descent & & & 0.20 \\
Age & 0.20 & $0.04^{*}$ & \\
Preop SCT - descent & 0.28 & 0.13 & \\
'P $<0.05, \dagger P<0.01$ & & &
\end{tabular}

